\title{
论文
}

\section{表层土壤孢粉组合的空间均质化可提高气候 预测的可靠性}

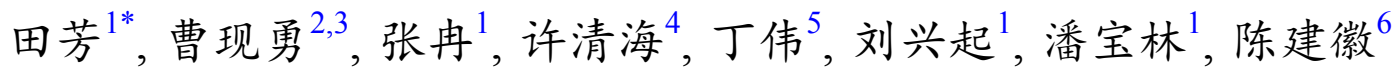

1. 首都师范大学资源环境与旅游学院, 北京 100048 ;

2. 中国科学院青藏高原研究所高寒生态重点实验室古生态与人类适应团队, 北京 100101 ;

3. 中国科学院青藏高原地球科学卓越创新中心, 北京 100101 ;

4. 河北师范大学资源与环境科学学院, 石家庄 050024 ;

5. Institute of Geological Sciences, Palaeontology, Free University of Berlin, Berlin 12249, Germany;

6. 兰州大学资源环境学院西部环境教育部重点实验室, 兰州 730000

* 通讯作者, E-mail: tianfang@cnu.edu.cn

收稿日期：2019-05-28; 收修改稿日期：2020-04-16; 接受日期：2020-06-09; 网络版发表日期：2020-07-30 国家自然科学基金项目(批准号: 41877459、41630753)、中国科学院率先行动“百人计划”项目、国家自然科学基金委员会与德国科学基金会 合作研究项目(编号: 41861134030)资助

摘要提高基于表土孢粉数据开展古气候重建可靠性的关键在于缩小其估测误差, 而表土孢粉组合易受局地植 物群落成分影响, 是估测误差较高(相对于湖泊表层沉积物孢粉组合)的原因之一. 本研究对东亚现代孢粉数据集 (含 $68 \%$ 表土样品)进行不同空间尺度均质化处理(孢粉组合百分含量的区域平均), 使用典型相关分析(CCA)和偏 最小二乘加权平均法(WA-PLS) 评估孢粉与气候关系及模型可靠性, 并选取区域性孢粉-降水校准集估测中国北 方季风尾问区湖泊表层沉积物孢粉组合的现代年降水, 以评估其估测能力. 结果表明, 空间均质化处理可以增加 CCA分析中气候变量对孢粉空间分布特征的解释量, 并改善孢粉-气候WA-PLS转换函数的交叉检验结果. 结果也 证实较小空间尺度(如半径为2、5及 $10 \mathrm{~km}$ ) 空间均质化处理可削弱表土孢粉组合中局地狍粉的干扰并提高交叉检 验结果, 而当空间尺度超过半径 $20 \mathrm{~km}$ 时会出现过度均质化现象, 干扰孢粉-气候关系. 此外, 本研究还发现湖泊边 缘表层沉积物孢粉组合易受河流远距离搬运而来的孢粉或湖泊边滩局地植物孢粉的影响, 其对区域性气候特征 的代表性弱于湖泊中心深水区的孢粉组合.

关键词中国北方, 表土孢粉, 气候重建, WA-PLS

\section{1 引言}

利用狍粉开展过去气候重建研究中，选取沉积环
境与地层狍粉组合一致的现代孢粉组合构建孢粉-气 候校准集, 可削弱狍粉来源范围及埋藏环境等差异带 来的估测误差，进而提高过去气候重建的可靠性

$\begin{array}{ll}\text { 中文引用格式: } & \text { 田芳, 曹现勇, 张由, 许清海, 丁伟, 刘兴起, 潘宝林, 陈建徽. 2020. 表层土壤狍粉组合的空间均质化可提高气候预测的可靠性. 中国科学: 地球 } \\ & \text { 科学, 50(10): 1468-1476, doi: 10.1360/N072019-0119 } \\ \text { 英文引用格式: } & \text { Tian F, Cao X, Zhang R, Xu Q, Ding W, Liu X, Pan B, Chen J. 2020. Spatial homogenization of soil-surface pollen assemblages improves the } \\ & \text { reliability of pollen-climate calibration-set. Science China Earth Sciences, https://doi.org/10.1007/s11430-019-9643-0 }\end{array}$ 
(Birks, 1995; Juggins和Birks, 2012). 湖泊沉积物具有 沉降速率稳定、年代序列可靠、沉积环境利于孢粉 保存等优点, 是利用狍粉开展过去气候及环境重建的 理想载体. 目前, 中国已开展了大量基于湖泊沉积物 的具有可靠年代序列和高时间分辨率的晚第四纪孢 粉研究(例如, Xiao等, 2014; Chen等, 2015; Zhang等, 2016; Wen等, 2017; Zhang等, 2018; Wu等, 2019; Xu 等, 2019), 对重建过去植被和气候具有重要的参考价 值. 然而，中国及东亚地区已发表的现代狍粉文献中, 只有少量孢粉数据采自于湖泊表层沉积物且其分布 范围局限于个别区域，如青藏高原(Shen等，2006; Herzschuh等，2010；Ma等，2017)和蒙古国西部等地 区(Tian等, 2014); 而中国东部地区湖泊较少, 现代狍 粉研究主要依赖于表层土壤和苔藓样品( $\mathrm{Ca}$ o 等, 2014), 影响了基于孢粉开展过去气候定量重建的可 靠性. 因此, 有必要评估基于表土现代孢粉重建过去 气候的可靠性, 以开展中国东部地区长时间尺度东亚 夏季风演变过程及机制研究(Zhao等，2009; Liu等, 2015).

已有研究表明, 基于加权平均的系列重建方法, 表土样品孢粉组合在交叉检验中易产生较高的估测 误差; 相比之下，湖泊表层沉积物狍粉组合的估测误 差较低(Cao等, 2014). 相对于湖泊表层沉积物狍粉组 合，表土狍粉组合易受局地群落成分和非目标环境因 素(如人类干扰、微地貌等)的影响(Zhao等, 2009), 进 而导致相同或相似的气候条件可能产生存在显著差 异的狍粉组合. 此外, 孢粉-气候校准集中孢粉样点的 气候数据通常是通过气象站数据空间插值(平滑) 获 得，易造成局地狍粉组合与区域性气候变量之间的不 匹配现象. 现代孢粉数据的空间均匀化处理具有消除 这一不匹配现象的潜力，但其效果和可靠性尚未被 验证.

本研究以东亚大陆现代狍粉数据集(涵盖中国及 蒙古国; 包含 2559 个孢粉样点，其中 $68 \%$ 为表土样点; Cao等，2014)为研究对象，经不同空间尺度均质化处 理后用于构建狍粉-气候校准集, 并估算中国北方季风 尾问区4 个湖泊表层沉积物狍粉组合所蕴含的气候信 息, 以评估其狍粉-气候关系及其气候估测能力. 本研 究拟回答以下问题: (1) 孢粉数据空间均质化可否降低 孢粉-气候关系中的噪音, 提高气候重建的可靠性? (2) 表土狍粉组合是否可用于构建狍粉-气候校准集并重
建古气候? (3) 湖泊表层沉积物狍粉组合能否代表区 域气候?

\section{2 数据与方法}

\section{1 孢粉及气候数据}

东亚现代狍粉数据集(涵盖中国和蒙古国)包含 2559 个孢粉样点, 其中表土样点1748个、湖泊表层沉 积物样点 398 个、尘土及苔藓样点 373 个(图1), 以及 168 个主要狍粉类型(至少出现于 3 个样品且最大值大 于3\%; Cao等, 2014). 基于中国、蒙古国、西伯利亚南 部及邻近地区气象站月均温度和降水的多年平均数据 (10年或30年), 参照STRM $1 \mathrm{~km}$ 数字高程模型(Farr等, 2007), 使用平滑样条插值法(ANUSPLIN软件, 4.36版; Hancock和Hutchinson，2006) 获得 $1 \mathrm{~km} \times 1 \mathrm{~km}$ 栅格月均 温度和降水数据. 选取距狍粉样点最近栅格的气候数 据代表该样点气候数据, 并计算其年降水量 $\left(P_{\mathrm{ann}}\right)$ 、最 冷月平均温度 $\left(M t_{\mathrm{co}}\right)$ 和最热月平均温度 $\left(M t_{\mathrm{wa}}\right)$. 详细的 气候数据获取方法可见文献(Cao等, 2014). 将 3 个气候 指标作为单独环境因子进行典型相关分析 (CCA), 结 果发现 $P_{\mathrm{ann}}$ 对孢粉组合解释率 (5.1\%)高于 $M t_{\mathrm{co}}$ 和 $M t_{\mathrm{wa}}$ 的 解释率(分别为 $4.4 \%$ 和 $2.8 \%$ ), 表明 $P_{\mathrm{ann}}$ 对本数据集孢粉 空间分布影响最大(Cao等, 2014). 此外, 3 个气候因子 对孢粉空间分布较低的解释率表明存在更多环境因子 影响着本数据集孢粉空间分布, 比如, 复杂的气候系 统、多样的地理单元、高的植物物种多样性及复杂孢 粉样品类型等; 在过去的气候重建中需要谨慎 $(\mathrm{CaO}$ 等, 2018).

为了评估孢粉-气候校准集的估测能力, 本研究选 取中国北方季风尾问区 4 个湖泊共 106 个表层湖泊沉积 物孢粉组合用于估算这些湖泊的现代 $P_{\mathrm{ann}}$ (Tian等, 2008)：包括岱海37个孢粉样点(采用与现代狍粉样点 相同的气候插值方法所得的现代 $P_{\mathrm{ann}}$ 为 $380 \mathrm{~mm}$, 下 同)、红碱淖 30 个样点 $(410 \mathrm{~mm})$ 、囫目淖 22 个样点 $(430 \mathrm{~mm})$ 和对口淖 $(420 \mathrm{~mm}) 17$ 个样点.

\section{2 数据分析方法}

为减少局地植物群落对狍粉组合的影响, 本研究 对每个现代孢粉组合进行空间均质化处理. 首先，使 用R语言(3.6.0版; R Core Team, 2019)fields软件包 (9.6.1版; Nychka等，2019)中的rdist.earth方程计算每 


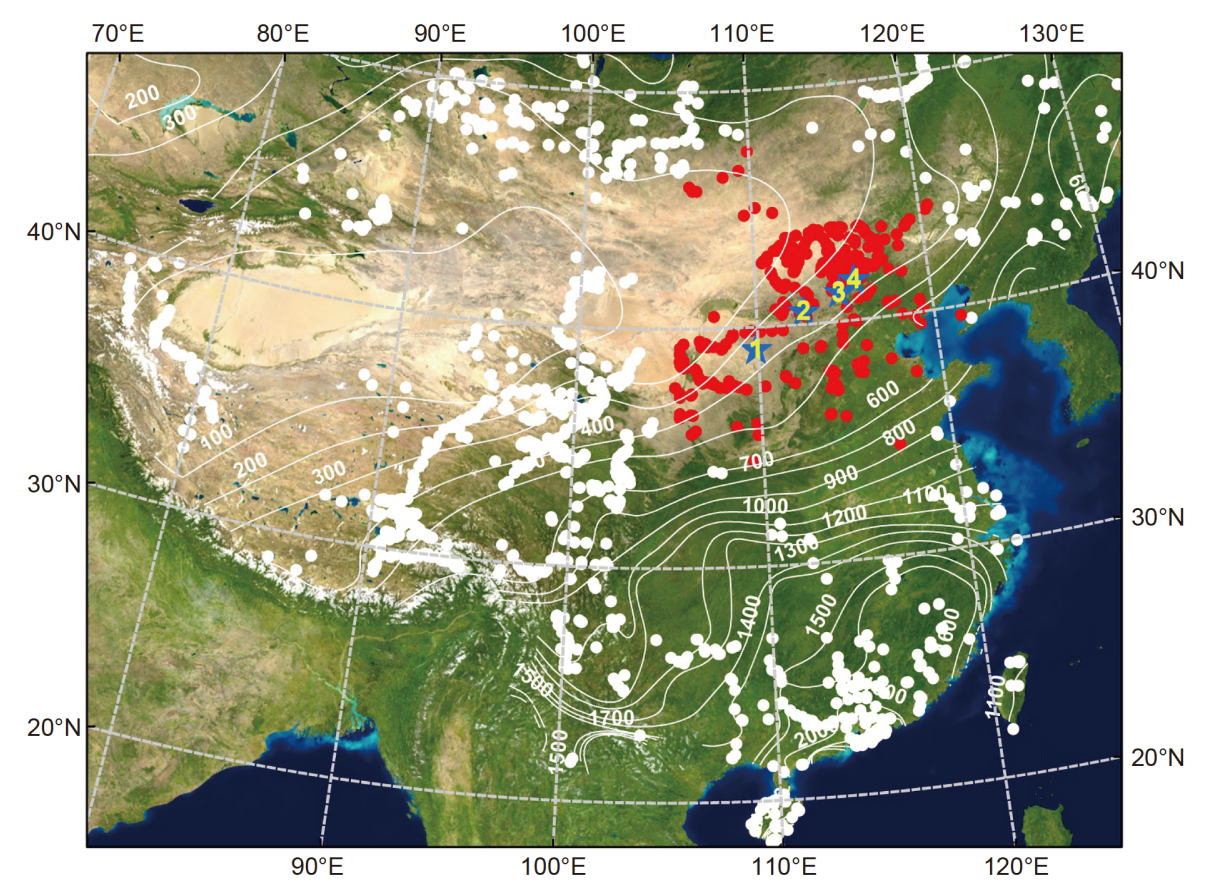

图 1 东亚现代狍粉数据集狍粉样点(白色圆点)及用于估算 4 个湖泊现代年降水的狍粉样点(红色圆点)分布

蓝色五星代表 4 个湖泊位置: 1 , 红碱淖; 2 , 岱海; 3 , 对口淖; 4 , 囫图淖. $P_{\text {ann }}$ 等值线是基于东亚 2015 个气象站数据利用克里格法插值获得

对狍粉样点(基于经度/纬度坐标)间的空间距离(球面 距离, $\mathrm{km}$ ), 产生样点间空间距离矩阵; 而后, 计算每个 样点周围不同距离内 $(2 、 5 、 10 、 20 、 30 、 60 、 90 、$ $120 \mathrm{~km}$ ) 所有样点原始狍粉百分比数据(包括该样点的 数据)的平均值, 作为该样点空间均质化处理后的狍粉 含量数据. 以 $2 \mathrm{~km}$ 均质化为例, 在目标样点 $\mathrm{a}$ 周围 $2 \mathrm{~km}$ 半 径内有 4 个孢粉样点 $(\mathrm{b} \sim \mathrm{e})$, 样点 $\mathrm{a}$ 的均质化孢粉百分含 量为 5 个样点 $(\mathrm{a} \sim \mathrm{e})$ 原始狍粉百分比的平均值; 而对于 目标样点 $b$, 只有样点 $\mathrm{a} 、 \mathrm{c} 、 \mathrm{~d}$ 出现在其 $2 \mathrm{~km}$ 范围内, 样 点 $\mathrm{b}$ 的均质化狍粉数据即是样点 $\mathrm{a} \sim \mathrm{d}$ 原始孢粉百分比的 平均值. 以此类推, 逐一对本现代狍粉数据集中 2559 个 样点孢粉百分比进行空间均质化处理; 但由于缺少邻 近样点，仍有部分样点无法被均质化处理. 经不同空 间尺度均质化处理后，孢粉数据可建立一系列具有相 同样点数量、相同狍粉类型的孢粉-气候校准集 $(n=8)$, 用于分析不同空间尺度均质化对狍粉-气候关系及定 量重建可靠性的影响.

将 168 个孢粉类型含量数据进行平方根转换, 并应 用于典型相关分析(CCA), 以分析不同空间尺度均质 化对孢粉-气候关系的影响. 分别将 3 个气候因子作为 单独环境限制因子加入CCA模型，并分别计算约束特
征值与第一个非约束特征值的比值 $\left(\lambda_{1} / \lambda_{2}\right)$, 比值越高 意味着气候因子对孢粉空间分布的影响越大(Juggins, 2013). CCA分析使用vegan程序包中的 $c c a$ 方程完成 (2.5-4版; Oksanen等, 2019).

为分析不同空间尺度均质化对校准集气候重建可 靠性的影响, 将leave-one-out交叉验证模型性能(包括 观测值和估测值之间相关系数, $r^{2}$; 估测均方根误差, RMSEP). 将平方根转换后的孢粉数据用于构建转化 函数, 以减弱数据的信噪比(Prentice, 1980). 已有研究 证实, 相对于现代类比法(MAT), WA-PLS法估测的可 靠性较高, 更适合于本东亚现代狍粉数据集(Cao等, 2014). 本研究采用WA-PLS法建立转换函数(rioja程序 包，0.9-15版; Juggins，2017), 并通过随机 $t$ 检验确定 WA-PLS成分(Juggins和Birks, 2012). 由于 $P_{\mathrm{ann}}$ 对整个数 据集孢粉空间分布影响最大(Cao等, 2014), 且已开展 的中国北方区域性孢粉-气候校准集研究也认为 $P_{\mathrm{ann}}$ 是 最重要的气候变量 $(X u$ 等, 2010; Chen等, 2015), 因此本 研究选择 $P_{\mathrm{ann}}$ 作为目标气候变量. 为检验空间均质化对 转换函数估测能力的影响, 本研究选择岱海周围 $800 \mathrm{~km}$ 范围内 $P_{\mathrm{ann}}$ 小于 $800 \mathrm{~mm}$ 的现代狍粉样点(图1中 红点), 基于原始狍粉百分比和被不同空间尺度均质化 
处理后的孢粉含量数据建立狍粉- $P_{\mathrm{ann}}$ 校准集(方法参 考: Chen等, 2015; Cao等, 2017; 陈建徽等, 2018); 并估 算采自于 4 个湖泊不同位置的 106 个表层沉积物孢粉组 合所蕴含的 $P_{\mathrm{ann}}$ 信息.

\section{3 结果}

\section{1 空间均质化对狍粉-气候关系的影响}

CCA 结果表明, 空间均质化处理显著提高了 3 个气 候因子对孢粉空间分布的解释率. $P_{\mathrm{ann}}$ 对孢粉数据空间 分布特征的解释率从原始狍粉数据集(未开展空间均 质化处理)的 $5.1 \%$ 升高到 $120 \mathrm{~km}$ 尺度均质化处理数据 的 $20.9 \%$ ，其中最大增长率出现在较小空间尺度均质 化(如半径 $2 、 5$ 和 $10 \mathrm{~km}$ )的狍粉数据集. 此外, $P_{\mathrm{ann}}$ 作为 单独环境控制因子获得的 $\lambda_{1} / \lambda_{2}$ 比值也显著升高，其中 经 $2 \mathrm{~km}$ 均质化处理的狍粉数据集的 $\lambda_{1} / \lambda_{2}$ 比值增幅最大 (图2). 总之, 局地尺度均质化处理可降低局地植物群 落成分对狍粉数据的影响，并增强表土孢粉组合对气 候的指示意义(网络版附表S1, http://earthen.scichina. com).

\section{2 空间均质化处理对孢粉-气候校准集交叉检验 的影响}

作为影响狍粉数据空间分布特征最重要的气候因 子，本研究基于原始的和经不同空间尺度均质化处理 后的狍粉数据集构建狍粉 $-P_{\mathrm{ann}}$ 转换函数. 相对于原始 孢粉数据集, $2 \mathrm{~km}$ 均质化的数据集模型的leave-one-out 交叉验证结果有明显改善, 表现为 $r^{2}$ 由 0.87 升高到 0.90 ,

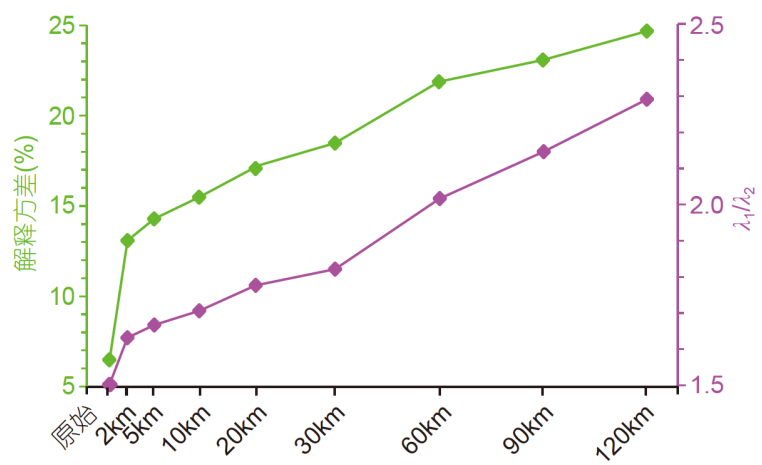

图 2 不同空间尺度均质化处理对 $P_{\mathrm{ann}}$ 对孢粉数据空间分 布解释量(绿色折线)和 $\lambda_{1} / \lambda_{2}$ 比值(紫色折线)的影响 由典型相关分析CCA获取
RMSEP从 $200 \mathrm{~mm}$ 降到 $175 \mathrm{~mm}$. 随着均质化处理空间尺 度的不断增大, 模型性能不断提高, 但提高的速率变慢 (图3; 附表S2).

基于原始狍粉数据转换函数交叉检验获得的估 测 $P_{\mathrm{ann}}$ 值与相对应的观测值存在一定偏差(图3). 一些 具有相似观测 $P_{\mathrm{ann}}$ 值的样点(例如位于神农架和台湾省 佩山的观测 $P_{\mathrm{ann}}$ 分别为 1100 和 $1200 \mathrm{~mm}$ 的样点)被估测 出差异显著的 $P_{\mathrm{ann}}$ 值, 表明狍粉组合受到垂直植被分布 的影响, 相似的 $P_{\mathrm{ann}}$ 可产生显著差异的孢粉组合; 然而 $2 \mathrm{~km}$ 尺度空间均质化可解决该问题(图3). 中等和较大 空间尺度的均质化 $(\geq 20 \mathrm{~km})$ 则导致另一个问题：具不 同观测 $P_{\mathrm{ann}}$ 值样点经过均质化处理后产生了相似的估 测 $P_{\mathrm{ann}}$ 值, 这表明狍粉数据被过度均质化.

\section{3 孢粉-气候校准集估测能力}

岱海周围 $800 \mathrm{~km}$ 范围内且观测 $P_{\mathrm{ann}}$ 低于 $800 \mathrm{~mm}$ 的 现代狍粉样点共有 474 个, 包括 447 个表土样品和 27 个 湖泊表层沉积物样品. 选择该 474 个样点的原始孢粉 数据以及经过 $2 、 5$ 和 $10 \mathrm{~km}$ 尺度均质化处理后的狍粉 数据分别构建WA-PLS转换函数. 由于 3 个经均质化处 理数据构建的转换函数具有相似的交叉验证结果, 且 得到106个表层湖泊沉积物孢粉组合相似的 $P_{\mathrm{ann}}$ 重建 结果(附图 $\mathrm{S} 1$ 和 $\mathrm{S} 2$ ), 本文只选用了 $5 \mathrm{~km}$ 尺度均质化数据 构建的转换函数与原始数据构建的转换函数进行对 比. 基于均质化数据的转换函数比基于原始数据的转 换函数具有更高的 $r^{2}$ (分别为 0.68 和 0.59 ) 和更低的 RMSEP值(分别为 87 和 $99 \mathrm{~mm}$; 图4).

对比发现, 由 $5 \mathrm{~km}$ 尺度均质化数据构建的转换函 数估测的 106 个湖泊表层沉积物孢粉组合的 $P_{\mathrm{ann}}$ 值略 低于由原始现代狍粉数据估测的结果(图5). 本文仅描 述和讨论由均质化转换函数估测的 $P_{\mathrm{ann}}$ 结果. 对于岱海 的 37 个样点, 估测 $P_{\mathrm{ann}}$ 和观测 $P_{\mathrm{ann}}$ 的差值在 $-58 \sim 118 \mathrm{~mm}$ 间波动; 且两者间差值超过 $50 \mathrm{~mm}$ (包括高估和低估)的 10 个样点中的 8 个样点位于湖泊边缘(图6). 红碱淖的 30 个样点的估测 $P_{\mathrm{ann}}$ 与观测 $P_{\mathrm{ann}}$ 差值的变幅比岱海略 小 $(-77 \sim 83 \mathrm{~mm})$, 而差值超过 $50 \mathrm{~mm}$ 的 6 个样点中有 5 个 位于湖岸附近. 两个较大湖泊的估测 $P_{\mathrm{ann}}$ 平均值略高 于观测值(岱海约高 $17 \mathrm{~mm}$, 红碱淖约高 $11 \mathrm{~mm}$; 图5). 两 个小型湖泊(囫目淖和对口淖)的估测 $P_{\mathrm{ann}}$ 平均值接近 于观测值, 且估测 $P_{\mathrm{ann}}$ 和观测 $P_{\mathrm{ann}}$ 差值波动范围也明显 低于两个较大湖泊(囫圈淖: $-36 \sim 70 \mathrm{~mm}$; 对口淖: 

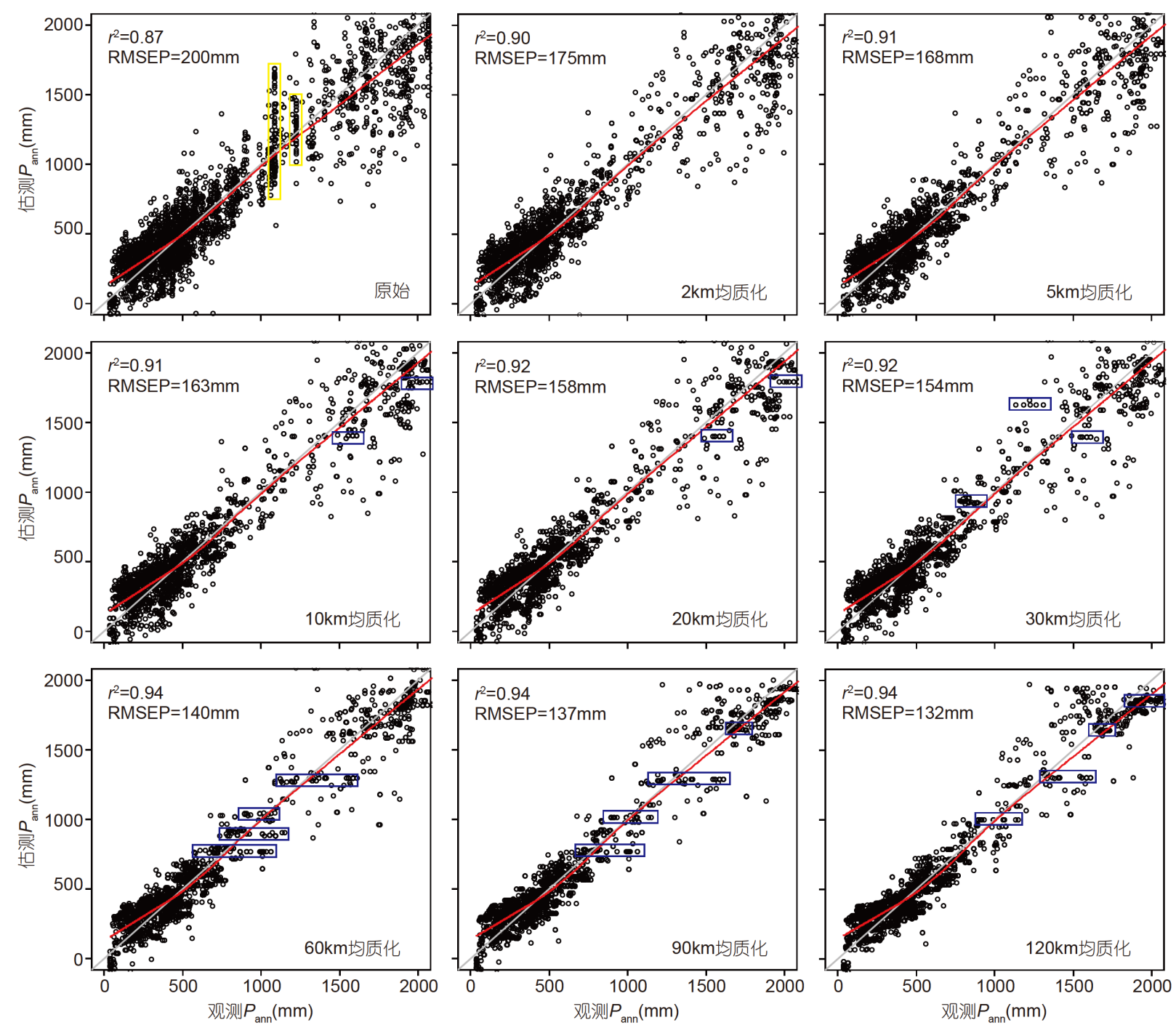

图 3 不同空间尺度均质化对偏最小二乘加权平均法(WA-PLS)leave-one-out交叉检验结果的影响

$-79 \sim 45 \mathrm{~mm}$ ). 两个小型湖泊共有 6 个样点产生了大于 $50 \mathrm{~mm}$ 的差值( 4 个出现在囫囵淖、 2 个出现在对口淖), 全部位于近湖岸或河流入湖口地带(图6).

\section{4 讨论}

\section{1 表土狍粉数据在气候重建中的潜力}

前人研究通常认为表层土壤中的孢粉主要来源于 样点周边狭小范围内(Jackson和Kearsley, 1998; Sugita 等, 1999), 其狍粉组合应代表局地植物群落组成( $\mathrm{Li}$ 等, 2009; Chen等, 2017; Huang等, 2018); 换言之, 局地成 分对表土狍粉组成的影响会导致其无法很好地反映区
域气候特征. 由于孢粉来源范围、传播途径及保存状 况等方面的差异(Tian等，2008; Xu等，2012)，表土孢 粉组合并不是开展湖泊沉积物孢粉组合古气候重建的 最优选择(Juggins和Birks，2012).Cao等(2014)发现相 对于湖泊表层沉积物狍粉组合, 表土孢粉组合在leaveone-out交叉验证中产生更高的RMSEP，表明表土孢粉 组合在指示区域气候方面存在较大的噪音.

区域土地覆被定量重建研究中，通过整合多个受 局地植被影响较强烈的泥炭或小型湖泊孢粉组合，可 削弱局地植物成分对狍粉组合的影响进而使其能够较 好地代表区域性植被特征(Sugita，2007; Mazier等, 2012). 这一做法表明, 孢粉数据的空间均质化可以改 

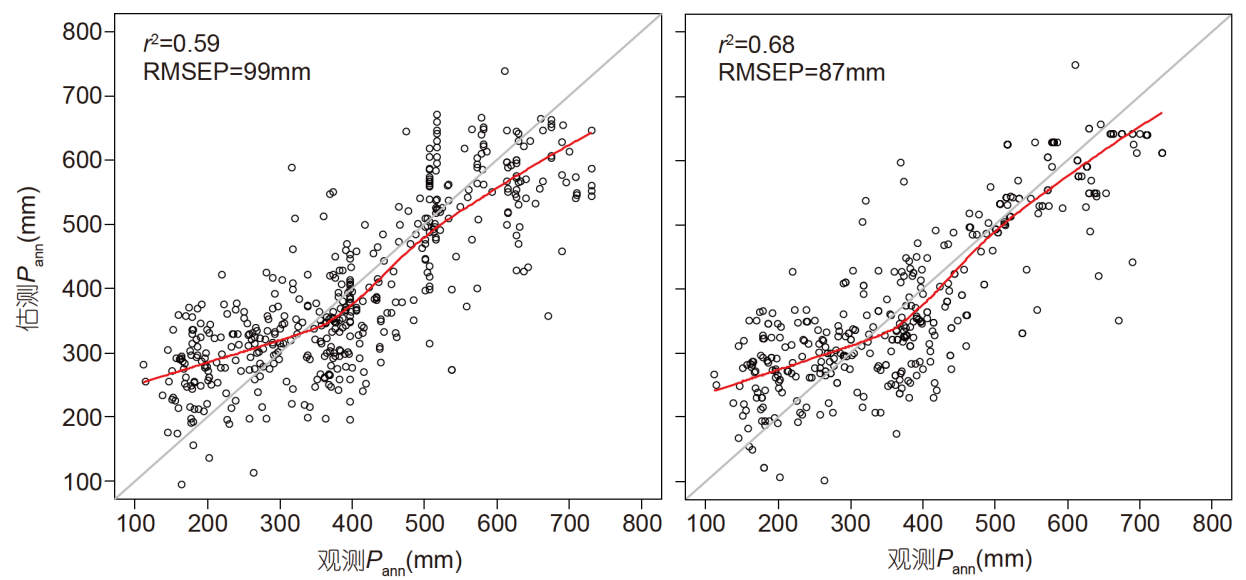

图 4 利用中国北方 474 个现代狍粉数据构建偏最小二乘加权平均法(WA-PLS)转换函数的leave-one-out交叉检验结果 左图为基于原始孢粉数据的结果, 右图为基于 $5 \mathrm{~km}$ 尺度空间均质化处理后狍粉数据的结果
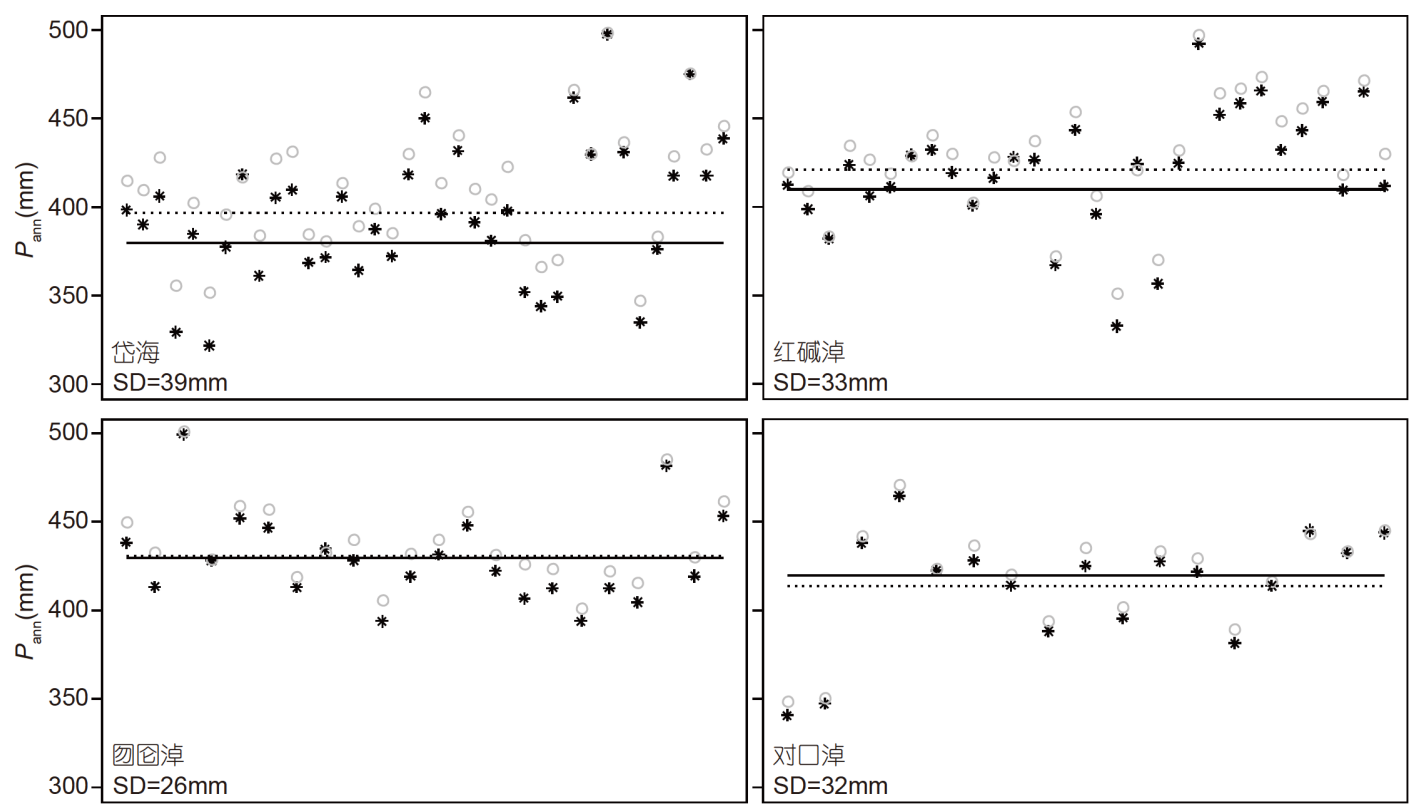

图 5 基于 474 个现代狍粉数据估算的中国北方 4 个湖泊现代 $P_{\mathrm{ann}}$ 结果

胞粉数据包括原始数据和 $5 \mathrm{~km}$ 尺度空间均质化数据. 水平实线代表 4 个湖泊基于插值法获取的 $P_{\text {ann }}$; 星形点、水平虚线和 $\mathrm{SD}$ 分别代表由 $5 \mathrm{~km}$ 空 间尺度均质化㘧粉数据估测的 $P_{\mathrm{ann}}$ 及其平均值和标准差; 圆形点代表由原始狍粉数据估测的 $P_{\mathrm{ann}}$

善表土狍粉组合对区域气候特征的指示意义. 本文系 统研究了不同空间尺度均质化处理对孢粉-气候关系 的影响. 结果表明, 较小尺度(如 $2 、 5$ 和 $10 \mathrm{~km}$ )均质化处 理可以降低局地植被带来的噪音并提高交叉检验的准 确性; 而大尺度(如大于 $20 \mathrm{~km}$ )均质化处理会扰乱孢粉 与气候的关系(图2和 3$)$. 总之, 应谨慎开展孢粉数据空 间均质化处理，特别是在植物成分存在显著差异的植 被交错带, 如中国北方和青藏高原东部的森林-草地交
错带. 此外, 本文中提到的最适宜的均质化尺度(如2、 5 和 $10 \mathrm{~km}$ )可能并不适用于其他地区孢粉数据, 而其他 现代狍粉数据集最适宜的均质化尺度应由具体分析 确定.

\section{2 由表土孢粉数据组建的校准集可用于古气候} 重建

本研究证实了表土狍粉数据(包括原始数据和均 


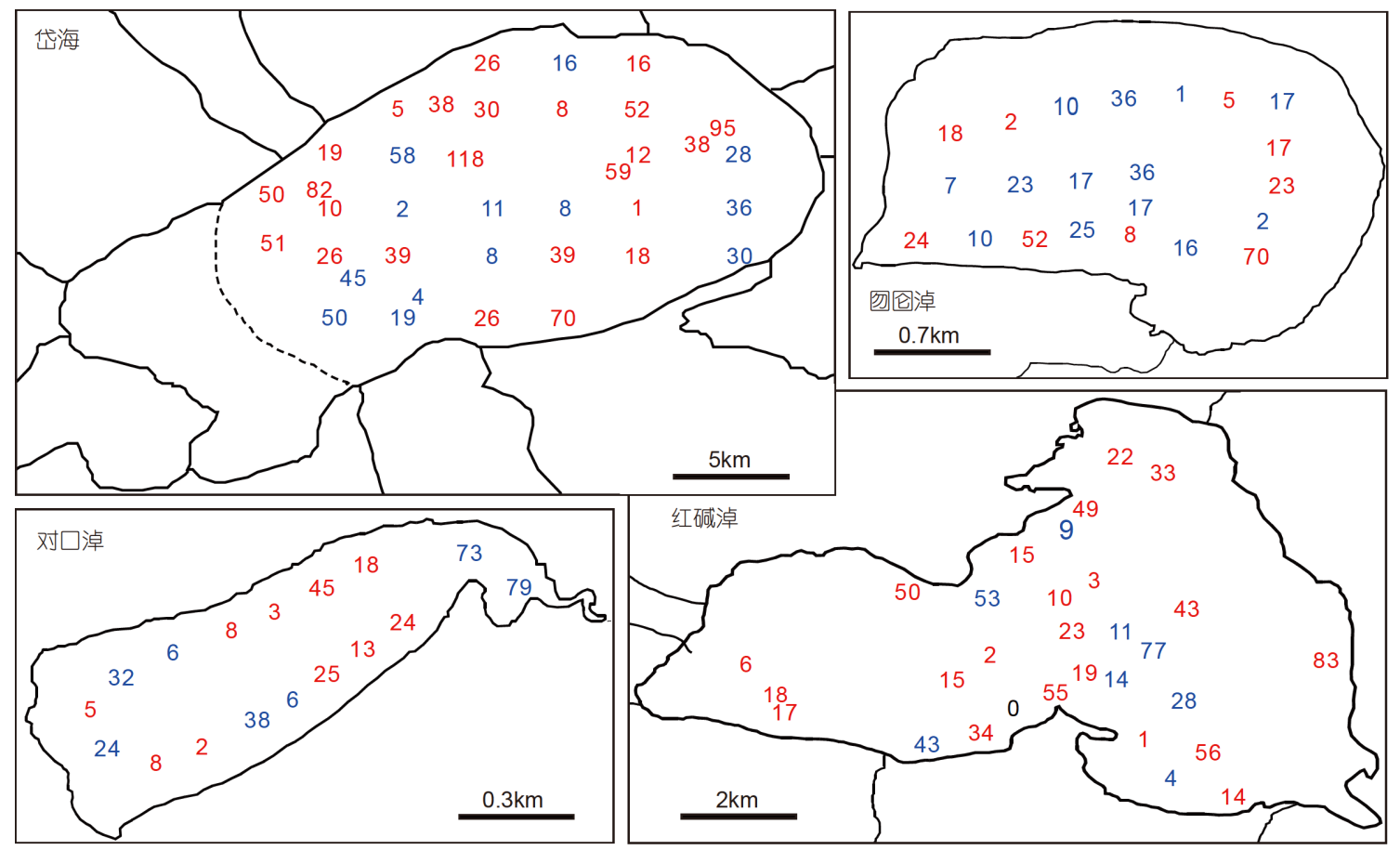

图 6 湖泊内 $\boldsymbol{P}_{\mathrm{ann}}$ 被高估(红色为正值)和被低估(蓝色为负值)狍粉样点空间分布

质化处理的数据)可用于重建古气候, 且具可靠的估测 能力; 而空间均质化处理可提高模型的可靠性. 采自 4 个湖泊的 106 个湖泊表层沉积物孢粉组合的 $P_{\mathrm{ann}}$ 估测 值与观测值较一致, 特别是经空间均质化处理后狍粉气候校准集的估测结果更接近于其观测值. 两个较大 型湖泊(岱海和红碱淖)现代狍粉组合估测 $P_{\mathrm{ann}}$ 的平均 值略高于两个湖泊由空间插值获得的 $P_{\mathrm{ann}}$, 而两个小型 湖泊基于孢粉估算的 $P_{\mathrm{ann}}$ 均值与其观测值存在轻微偏 差(图5). 此外，中国北方季风尾闾区的4个湖泊基于气 象站观测数据空间插值获得的 $P_{\mathrm{ann}}$ 为 $380 \sim 430 \mathrm{~mm}$; 为 此本研究选取中国北方 $P_{\mathrm{ann}}$ 梯度为 100 800 $\mathrm{mm}$ 的现代 孢粉数据集构建狍粉-气候校准集, 确保目标孢粉组合 $P_{\mathrm{ann}}$ 位于校准集 $P_{\mathrm{ann}}$ 梯度的中心，以减弱加权平均系列 重建方法“边缘效应”的影响(Birks, 1998); 表土狍粉数 据可靠的估测能力也在一定程度上与样点沿 $P_{\mathrm{ann}}$ 梯度 的均匀分布有关(Telford和Birks, 2011).

\section{3 湖泊表层狍粉组合对区域气候的代表性}

前人研究发现由河流携带而来的狍粉会增加湖泊 内部不同位置孢粉组合的差异性(如黄小忠等，2004; Xu等，2005; Wang等，2014), 进而导致气候重建误差
的增大. 基于湖泊表层沉积物狍粉组合估测的冬给措 纳湖年降水波动于 $320 \sim 402 \mathrm{~mm}$ ，而器测年降水为 $375 \mathrm{~mm}$ ，其中存在较大误差的表层样点主要位于湖泊 边缘地带(Wang等, 2014), 这一现象与本研究的结果 一致. 基于湖泊边缘和入湖河口附近沉积物狍粉组合 估测的现代气候与器测气候数据存在较大误差, 表明 由河流携带而来的狍粉以及湖岸局地植物狍粉对湖泊 孢粉组合影响较大. Tian等(2008)发现较大湖泊内部不 同位置狍粉组合差异性高于较小型湖泊，可能是湖泊 间的水深、孢粉来源范围、有无河流注入及流域内植 被等共同作用的结果. 湖泊深水区水体对狍粉沉积过 程的汇聚和混合效应较强, 较大型湖泊(如岱海和红碱 淖)在较深位置样品的孢粉组合一致性比较浅水区的 要好(Tian等, 2008), 可以解释本研究中较深位置孢粉 组合估测 $P_{\text {ann }}$ 更接近于观测值的现象. 由于两个小型湖 泊(囫囵湖和对口淖)湖水的混合效应更强，因此湖泊 内部狍粉组合差异性更小(Tian等, 2008), 可以解释较 小的气候重建误差. 此外, 现代孢粉组合高估了较大型 湖泊的 $P_{\mathrm{ann}}$, 这可能与较大型湖泊狍粉组合中较高的乔 木孢粉含量有关. 这些乔木孢粉可能来源于更湿润的 中国北部森林地区，通过风以及河流长距离运输传播 
沉积到湖泊沉积物中(Zhao等, 2009). 湖泊中部深水区 孢粉组合重建的气候存在较小误差, 能够更加准确的 反应本地区的气候.

\section{5 结论}

狍粉数据空间均质化处理可降低局地植物花粉对 孢粉组合的影响，在一定程度上提高其对区域气候的 指示意义，进而提高基于加权平均系列重建方法开展 气候重建的可靠性. 经适当空间尺度均质化处理的表 土孢粉组合可用于构建狍粉-气候校准集并具有很高 的可靠性. 此外, 位于湖泊边缘浅水区的孢粉组合易受 河流携带而来狍粉以及湖岸局地植物孢粉的影响，对 区域气候的代表性弱于湖泊中心部位深水区的孢粉组 合, 为此本研究建议利用孢粉开展古气候重建研究中 的湖泊沉积物应采自于湖泊中部深水区.

致谢文中的现代孢粉数据由国内外众多孢粉学者提 供, 作者在此谨致谢忱. 感谢审稿专家的宝贵建议.

\section{参考文献}

陈建徽, 吕飞亚, 黄小忠, Birks H J B, Telford R J, 张生瑞, 许清海, 赵 艳, 王海鹏, 周爱锋, 黄伟, 刘建宝, 魏国英. 2018. 基于孢粉的古气 候参数定量重建: 一种新思路及其在中国的应用实例. 中国科学: 地球科学, 48: 42-50

黄小忠, 赵艳, 程波, 陈发虎, 徐俊荣. 2004. 新疆博斯腾湖表层沉积 物的宝粉分析. 冰川冻土, 26: 602-609

Birks H J B. 1995. Quantitative palaeoenvironmental reconstructions. In: Maddy D, Brew J S, eds. Statistic Modelling of Quaternary Science Data. Cambridge: Quaternary Research Association. 161-254

Birks H J B. 1998. Numerical tools in palaeolimnology-progress, potentialities, and problems. J Paleolimnol, 20: 307-332

Cao X Y, Herzschuh U, Telford R J, Ni J. 2014. A modern pollenclimate dataset from China and Mongolia: Assessing its potential for climate reconstruction. Rev Palaeobot Palynol, 211: 87-96

Cao X, Tian F, Telford R J, Ni J, Xu Q, Chen F, Liu X, Stebich M, Zhao Y, Herzschuh U. 2017. Impacts of the spatial extent of pollenclimate calibration-set on the absolute values, range and trends of reconstructed Holocene precipitation. Quat Sci Rev, 178: 37-53

Cao X, Tian F, Ding W. 2018. Improving the quality of pollen-climate calibration-sets is the primary step for ensuring reliable climate reconstructions. Sci Bull, 63: 1317-1318

Chen F, Xu Q, Chen J, Birks H J B, Liu J, Zhang S, Jin L, An C, Telford
R J, Cao X, Wang Z, Zhang X, Selvaraj K, Lu H, Li Y, Zheng Z, Wang H, Zhou A, Dong G, Zhang J, Huang X, Bloemendal J, Rao Z. 2015. East Asian summer monsoon precipitation variability since the last deglaciation. Sci Rep, 5: 11186

Chen X, Huang X, Wu D, Zhang X, Dodson J, Zhou A, Chen F. 2017. Modern pollen assemblages in topsoil and surface sediments of the Xingyun Lake catchment, central Yunnan Plateau, China, and their implications for interpretation of the fossil pollen record. Rev Palaeobot Palynol, 241: 1-12

Farr T G, Rosen P A, Caro E, Crippen R, Duren R, Hensley S, Kobrick M, Paller M, Rodriguez E, Roth L, Seal D, Shaffer S, Shimada J, Umland J, Werner M, Oskin M, Burbank D, Alsdorf D. 2007. The shuttle radar topography mission. Rev Geophys, 45: RG2004

Herzschuh U, Birks H J B, Mischke S, Zhang C, Böhner J. 2010. A modern pollen-climate calibration set based on lake sediments from the Tibetan Plateau and its application to a Late Quaternary pollen record from the Qilian Mountains. J Biogeogr, 37: 752-766

Huang X, Chen X, Du X. 2018. Modern pollen assemblages from human-influenced vegetation in northwestern China and their relationship with vegetation and climate. Veget Hist Archaeobot, 27: 767-780

Hancock P A, Hutchinson M F. 2006. Spatial interpolation of large climate data sets using bivariate thin plate smoothing splines. Environ Model Software, 21: 1684-1694

Jackson S T, Kearsley J B. 1998. Quantitative representation of local forest composition in forest-floor pollen assemblages. J Ecol, 86: 474-490

Juggins S. 2017. rioja: Analysis of Quaternary Science Data. version 0.9-15. Available at: http://cran.r-project.org/web/packages/rioja/ index.html

Juggins S. 2013. Quantitative reconstructions in palaeolimnology: New paradigm or sick science. Quat Sci Rev, 64: 20-32

Juggins S, Birks H J B. 2012. Quantitative environmental reconstructions from biological data. In: Birks H J B, Lotter A F, Juggins S, Smol J P, eds. Tracking environmental change using lake sediments (vol 5), Data handling and numerical techniques. Dordrecht: Springer. 431-494

Li Y, Xu Q, Zhang L, Wang X, Cao X, Yang X. 2009. Modern pollen assemblages of the forest communities and their relationships with vegetation and climate in northern China. J Geogr Sci, 19: 643-659

Liu J B, Chen J H, Zhang X J, Li Y, Rao Z G, Chen F H. 2015. Holocene East Asian summer monsoon records in northern China and their inconsistency with Chinese stalagmite $\delta^{18} \mathrm{O}$ records. EarthSci Rev, 148: 194-208

Ma Q, Zhu L, Lu X, Wang Y, Guo Y, Wang J, Ju J, Peng P, Tang L. 2017. Modern pollen assemblages from surface lake sediments and 
their environmental implications on the southwestern Tibetan Plateau. Boreas, 46: 242-253

Mazier F, Gaillard M J, Kuneš P, Sugita S, Trondman A K, Broström A. 2012. Testing the effect of site selection and parameter setting on REVEALS-model estimates of plant abundance using the Czech Quaternary Palynological Database. Rev Palaeobot Palynol, 187: 38-49

Nychka D, Furrer R, Paige J, Sain S. 2019. fields: Tools for spatial data, version 9.6.1. Available at: https://cran.r-project.org/web/packages/ fields/

Oksanen J, Blanchet F G, Friendly M, Kindt R, Legendre P, McGlinn D, Minchin P R, O’Hara R B, Simpson G L, Solymos P, Stevens M H H, Szoecs E, Wagner H. 2019. vegan: Community Ecology Package, version 2.0-4. Available at: https://cran.r-project.org/web/packages/ vegan/index.html

Prentice I C. 1980. Multidimensional scaling as a research tool in Quaternary palynology: A review of theory and methods. Rev Palaeobot Palynol, 31: 71-104

R Core Team, 2019. R: A language and environment for statistical computing. R Foundation for Statistical Computing, Vienna

Shen C, Liu K, Tang L, Overpeck J T. 2006. Quantitative relationships between modern pollen rain and climate in the Tibetan Plateau. Rev Palaeobot Palynol, 140: 61-77

Sugita S. 2007. Theory of quantitative reconstruction of vegetation I: Pollen from large sites REVEALS regional vegetation composition. Holocene, 17: 229-241

Sugita S, Gaillard M J, Broström A. 1999. Landscape openness and pollen records: A simulation approach. Holocene, 9: 409-421

Telford R J, Birks H J B. 2011. Effect of uneven sampling along an environmental gradient on transfer-function performance. J Paleolimnol, 46: 99-106

Tian F, Herzschuh U, Telford R J, Mischke S, Van der Meeren T, Krengel M. 2014. A modern pollen-climate calibration set from central-western Mongolia and its application to a late glacialHolocene record. J Biogeogr, 41: 1909-1922

Tian F, Xu Q H, Li Y C, Cao X Y, Wang X L, Zhang L Y. 2008. Pollen assemblage characteristics of lakes in the monsoon fringe area of China. Sci Bull, 53: 3354-3363

Wang Y, Herzschuh U, Shumilovskikh L S, Mischke S, Birks H J B, Wischnewski J, Böhner J, Schlütz F, Lehmkuhl F, Diekmann B,
Wünnemann B, Zhang C. 2014. Quantitative reconstruction of precipitation changes on the NE Tibetan Plateau since the Last Glacial Maximum-extending the concept of pollen source area to pollen-based climate reconstructions from large lakes. Clim Past, 10: $21-39$

Wen R, Xiao J, Fan J, Zhang S, Yamagata H. 2017. Pollen evidence for a mid-Holocene East Asian summer monsoon maximum in northern China. Quat Sci Rev, 176: 29-35

Wu J, Liu Q, Cui Q Y, Xu D K, Wang L, Shen C M, Chu G Q, Liu J Q. 2019. Shrinkage of East Asia winter monsoon associated With increased ENSO events since the Mid-Holocene. J Geophys ResAtmos, 124: 3839-3848

Xiao X, Haberle S G, Shen J, Yang X, Han Y, Zhang E, Wang S. 2014. Latest Pleistocene and Holocene vegetation and climate history inferred from an alpine lacustrine record, northwestern Yunnan Province, southwestern China. Quat Sci Rev, 86: 35-48

Xu D, Lu H, Chu G, Liu L, Shen C, Li F, Wang C, Wu N. 2019. Synchronous 500-year oscillations of monsoon climate and human activity in Northeast Asia. Nat Commun, 10: 4105

Xu Q, Xiao J, Li Y, Tian F, Nakagawa T. 2010. Pollen-based quantitative reconstruction of Holocene climate changes in the Daihai Lake area, Inner Mongolia, China. J Clim, 23: 2856-2868

Xu Q, Li Y C, Yang X L, Xiao J L, Liang W D, Peng Y J. 2005. Source and distribution of pollen in the surface sediment of Daihai Lake, inner Mongolia. Quaternary Int, 136: 33-45

Xu Q, Tian F, Bunting M J, Li Y, Ding W, Cao X, He Z. 2012. Pollen source areas of lakes with inflowing rivers: Modern pollen influx data from Lake Baiyangdian, China. Quat Sci Rev, 37: 81-91

Zhang E, Wang Y, Sun W, Shen J. 2016. Holocene Asian monsoon evolution revealed by a pollen record from an alpine lake on the southeastern margin of the Qinghai-Tibetan Plateau, China. Clim Past, 12: 415-427

Zhang S, Xiao J, Xu Q, Wen R, Fan J, Huang Y, Yamagata H. 2018. Differential response of vegetation in Hulun Lake region at the northern margin of Asian summer monsoon to extreme cold events of the last deglaciation. Quat Sci Rev, 190: 57-65

Zhao Y, Xu Q, Huang X, Guo X, Tao S. 2009. Differences of modern pollen assemblages from lake sediments and surface soils in arid and semi-arid China and their significance for pollen-based quantitative climate reconstruction. Rev Palaeobot Palynol, 156: 519-524

(责任编委: 赵艳) 